\title{
Recent developments in Monte-Carlo Event Generators
}

\author{
Marek Schönherr ${ }^{1, a}$ \\ ${ }^{1}$ Institut für Theoretische Physik, Universität Zürich, 8057 Zürich, Switzerland
}

\begin{abstract}
With Run II of the LHC having started, the need for high precision theory predictions whose uncertainty matches that of the data to be taken necessitated a range of new developments in Monte-Carlo Event Generators. This talk will give an overview of the progress in recent years in the field and what can and cannot be expected from these newly written tools.
\end{abstract}

\section{Introduction}

Modern Monte-Carlo Event Generators like PyTHIA8 [1], HERWIG++ [2, 3] and SHERPA [4] are instrumental in most physics analyses and measurements at the LHC. The current state-of-the art in usage at the experiments are either next-to-leading order to parton shower matched calculations (NLOPS) or multijet merged ones at leading order accuracy. Examples for their widespread use are shown in Fig. 1. In many instances the PYTHIA8 and HERWIG++ generators (or their older predecessors) receive input from parton level tools computing the hard core production matrix elements either at NLO for processes with few final state particles (e.g. MADGRAPH5_AMC@NLO [5] or POWHEGBOX [6]), or at LO for multileg processes (e.g. ALPGEN [7] or MADGRAPH5_AMC@NLO). The following contribution highlights a few important improvements thereupon effected in recent years.

\section{Parton shower developments}

The first avenue improvements in event generators have been accomplished in recent years are parton showers. Being instrumental for the description of many relevant observables parton showers are a main ingredient of all event generator frameworks and thus their continuing advancement is crucial to a better description of collider observables.

On the one hand side subleading colour information has been propagated into the algorithms otherwise operating in the leading colour limit. In the first such advancement it was a pure necessity to achieve a process independent NLO matching and was consequently only introduced in the first emission [10]. Later implementations trace subleading colour information in different limits through multiple, if not all, emissions of the parton shower evolution [11, 12]. Generally, the impact of such improvements is small, as shown in Fig. 2 (left), although also highly sensitive observables exist [13].

Other works build around gaining a higher degree of analytical control over the parton showers' resummation properties [14]. Through the accompanying scrutiny also their predictive power and

\footnotetext{
a e-mail: marek.schoenherr@physik.uzh.ch
} 

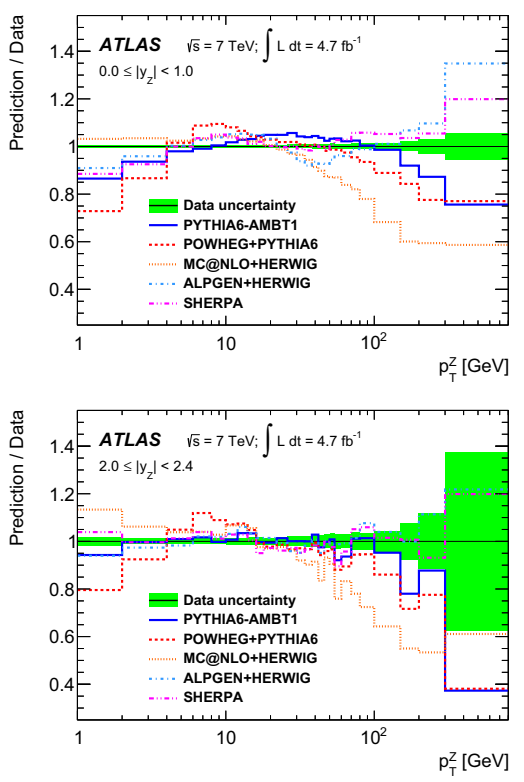

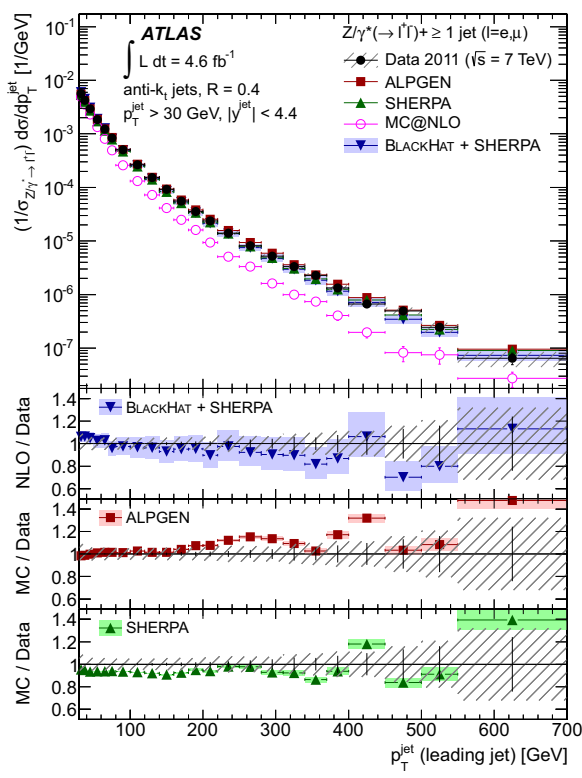

Figure 1. Left: Transverse momentum of the reconstructed $Z$ boson in the central and the forward region, as measured by the ATLAS detector. Figure taken from [8]. Right: Transverse momentum of the leading jet in $Z$ boson production in association with jets, as measured by the ATLAS detector. Figure taken from [9].
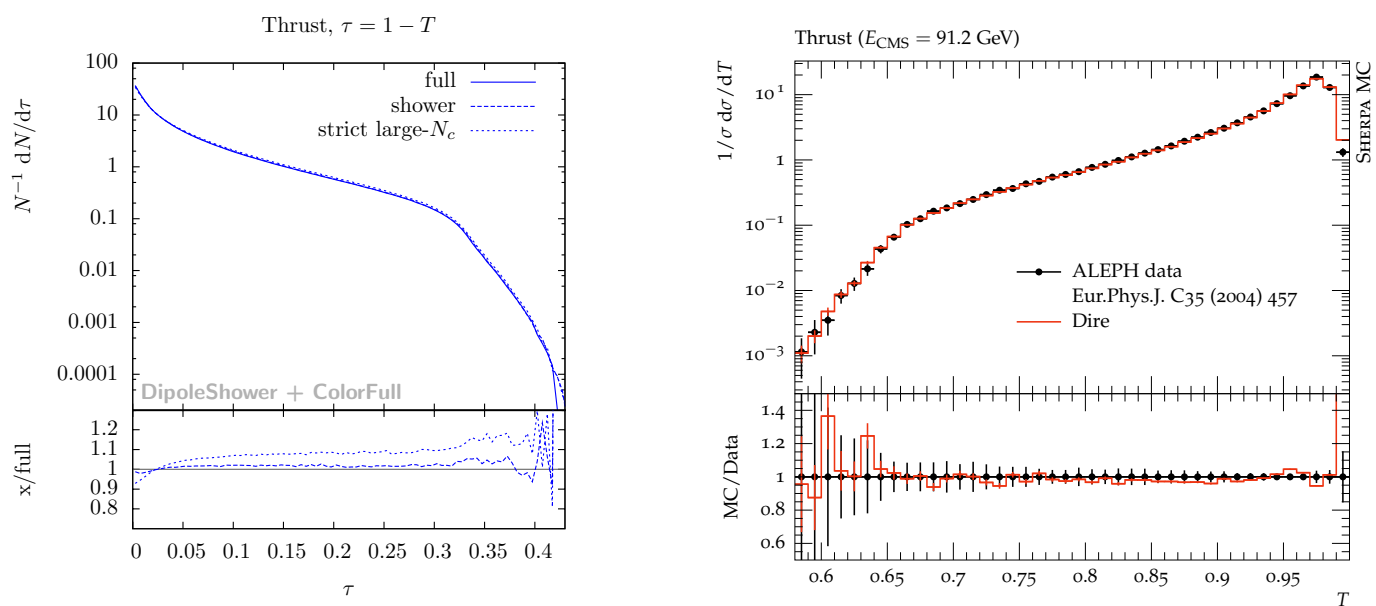

Figure 2. Left: Subleading colour effects in parton shower evolution in thrust in $e^{+} e^{-}$-collisions at LEP. Figure taken from [11]. Right: Thrust in $e^{+} e^{-}$-collisions at LEP as calculated by a new dipole shower implementation DIRE. Figure taken from [14].

ability to describe data has been improved. Fig. 2 (right) details the results of the newly written DIRE parton shower as compared to ALEPH data. 

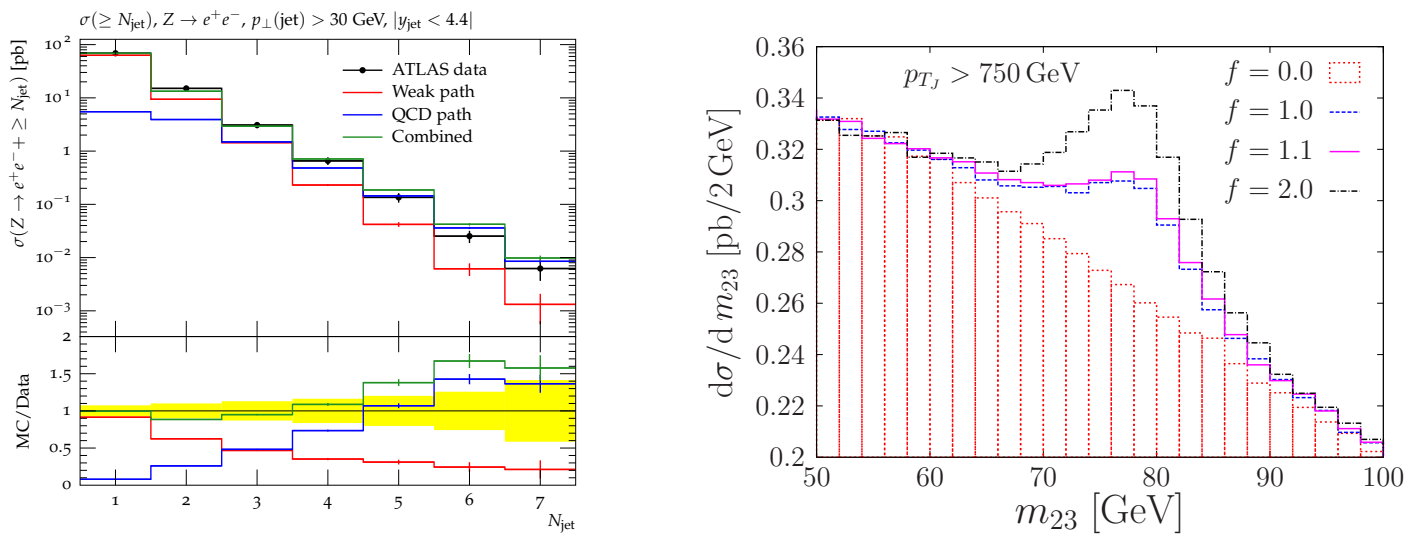

Figure 3. Left: Interplay of QCD evolution on top of $W$ production and EW evolution on top of jet production in describing $W$ plus mulitjet production. Figure taken from [15]. Right: Effects of adding EW evolution on subjet invariant masses. Figure taken from [16].

The third stream of development centres around incorporating electroweak effects into parton showers [15-17]. The emission of $W$ and $Z$ bosons, although rare, can be an important ingredient, especially in the highly boosted regime. Fig. 3 such effects for various observables. Such softcollinear approximations to higher-order electroweak corrections complement the approximate NLO electroweak corrections of [18] and the recently achieved automation of NLO electroweak corrections [19-21].

\section{NLOPs matching}

Known under the names of MC@ NLO [22] and PowHEG [23, 24], methods for matching NLO computations to parton showers are around for over ten years now. Recent years have seen small theoretical improvements on both schemes that lead to their application to a wider range of processes [10, 25-27] with a more complicated internal structure. The range of showers the respective matching schemes are available for has increased likewise [2, 3, 28, 29]. An systematically different matching method, UNLOPS, was developed in [30].

Similarly, CKKW [33] method of scale setting and Sudakov factor inclusion has been elevated to be applicable to NLO QCD computations in [34], leading to an improvement of NLOPs matched computations incorporating jets in the final state already at Born level. In colour singlet production in association with one additional jet the inclusion of a proper process dependent finite term can restore NLO accuracy for inclusive singlet production as well [35]. This formed the basis for the development of a NNLOPS matching method for colour singlet production [31,36]. An exemplary result is shown in Fig. 4 (left). Another NNLOPs matching scheme basing basing on MC@NLO and UNLOPs matching was developed for the same process class in $[32,37]$. Fig. 4 (right) details the results for this scheme named $\mathrm{UN}^{2} \mathrm{LOPS}$.

\section{Multijet merging}

Multijet merging aims at consistently combining calculations for the production of a certain experimental signature, like lepton pairs, Higgs bosons or top quark pairs, in association with any number 

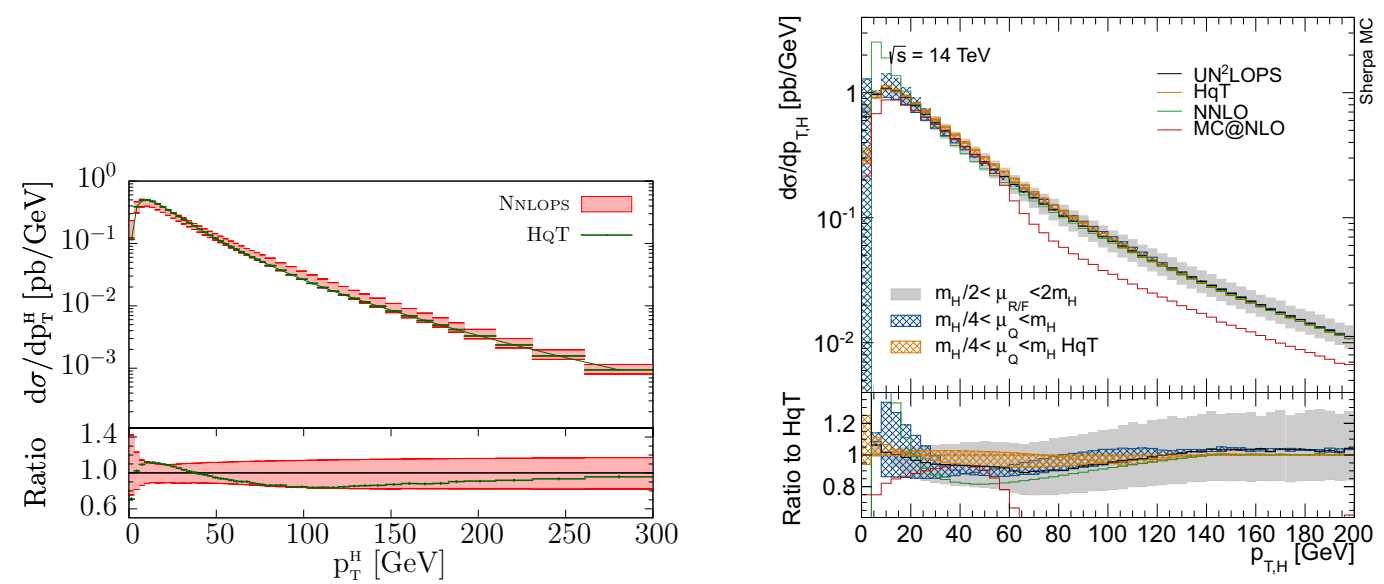

Figure 4. Left: Transverse momentum of the Higgs boson described at NNLOPS in the MiNLO approach. Figure taken from [31]. Right: Transverse momentum of the Higgs boson described at NNLOPS in the UN ${ }^{2}$ LOPS approach. Figure taken from [32].

of jets. As many observables do not clearly separate between different jet multiplicities but instead receive substantial contributions by e.g. one, two and three jet final states, such multijet merging schemes are the best way to calculate these observables with the highest accuracies.

At the NLO, this was pioneered in [40]. Modern implementations for hadron colliders first appeared as MEPs@NLo [41-43] and were applied to a wide range of processes [13, 39, 44-47]. Other implementations using other methods to calculated the matched processes for each jet multiplicity have been established in [38] and [30]. Fig. 5 details results of all three mentioned methods.

\section{Conclusions}

Monte-Carlo Event Generators are in good shape for Run II of the LHC. Tremendous progress in terms of the achieved accuracy in calculating the hard scattering process has been achieved. They can thus be used as for precise theoretical predictions including an evaluation of the theoretical uncertainty. Developments for the non-perturbative component of high-energy collisions, however, remain sparse. In that regime, playing a role in every hadron collider event, still phenomenologically motivated models with a large number of to-be-tuned parameters are instrumental in all generators. Thus, for precision calculations one should still try to minimise the influence of that regime on the considered observables.

MS acknowledges funding by the Swiss National Science Foundation (SNF) under contract PP00P2-128552.

\section{References}

[1] T. Sjöstrand, S. Ask, J.R. Christiansen, R. Corke, N. Desai, P. Ilten, S. Mrenna, S. Prestel, C.O. Rasmussen, P.Z. Skands, Comput. Phys. Commun. 191, 159 (2015), 1410. 3012

[2] J. Bellm et al. (2013), 1310.6877 

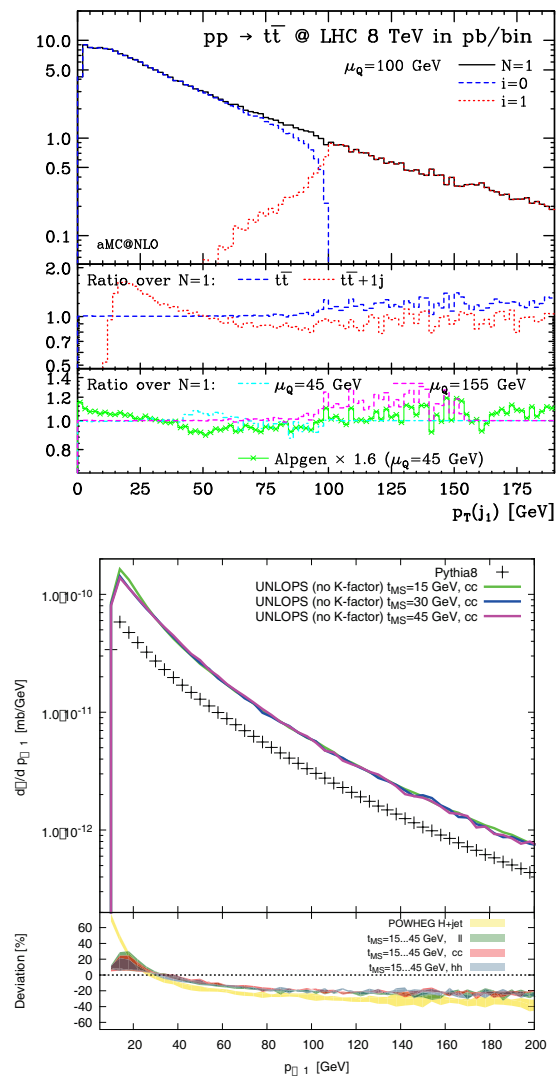

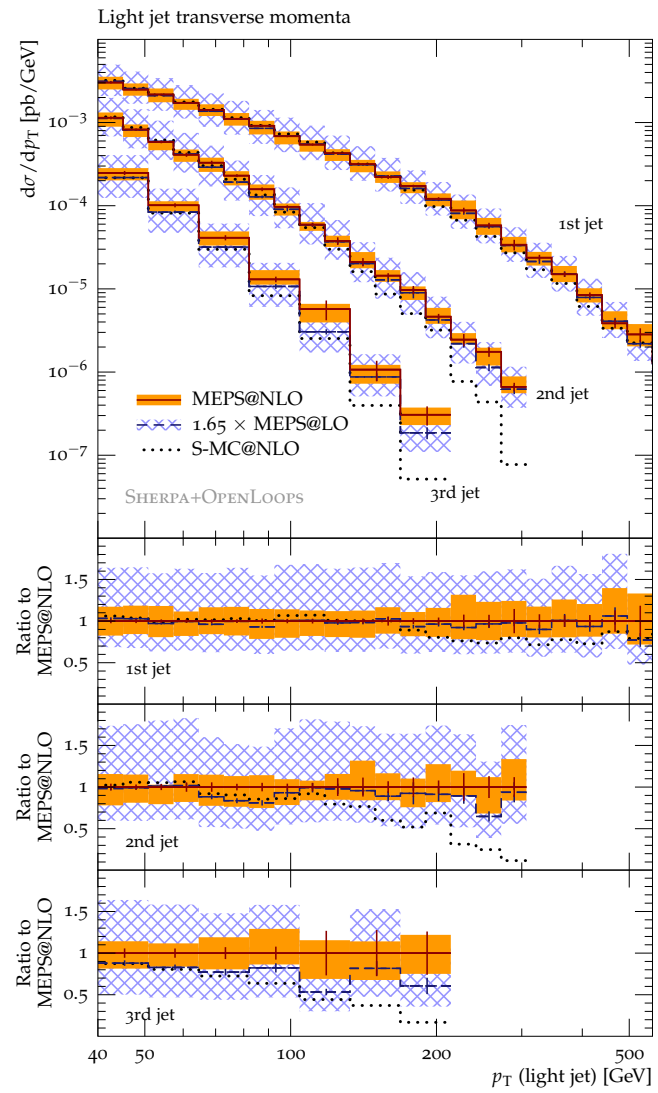

Figure 5. Left top: Transverse momentum of the leading jet in top pair production in association with jets described through a FXFX combination. Figure taken from [38]. Left bottom: Transverse momentum of the leading jet in Higgs production in association with jets described through UNLOPS merging. Figure taken from [30]. Right: Transverse momentum of the three leading jets in top pair production in association with jets described through MEPs@NLO merging. Figure taken from [39].

[3] J. Bellm et al. (2015), 1512.01178

[4] T. Gleisberg, S. Höche, F. Krauss, M. Schönherr, S. Schumann, F. Siegert, J. Winter, JHEP 02, 007 (2009), 0811. 4622

[5] J. Alwall, R. Frederix, S. Frixione, V. Hirschi, F. Maltoni, O. Mattelaer, H.S. Shao, T. Stelzer, P. Torrielli, M. Zaro, JHEP 07, 079 (2014), 1405.0301

[6] S. Alioli, P. Nason, C. Oleari, E. Re, JHEP 06, 043 (2010), 1002 . 2581

[7] M.L. Mangano, M. Moretti, F. Piccinini, R. Pittau, A.D. Polosa, JHEP 07, 001 (2003), hep-ph/0206293

[8] G. Aad et al. (ATLAS), JHEP 09, 145 (2014), 1406.3660

[9] G. Aad et al. (ATLAS), JHEP 07, 032 (2013), 1304 . 7098

[10] S. Höche, F. Krauss, M. Schönherr, F. Siegert, JHEP 09, 049 (2012), 1111.1220

[11] S. Plätzer, M. Sjödahl, JHEP 07, 042 (2012), 1201 . 0260

[12] Z. Nagy, D.E. Soper, JHEP 06, 097 (2014), 1401. 6364 
[13] S. Höche, J. Huang, G. Luisoni, M. Schönherr, J. Winter, Phys. Rev. D88, 014040 (2013), 1306.2703

[14] S. Höche, S. Prestel, Eur. Phys. J. C75, 461 (2015), 1506.05057

[15] J.R. Christiansen, T. Sjöstrand, JHEP 04, 115 (2014), 1401.5238

[16] F. Krauss, P. Petrov, M. Schönherr, M. Spannowsky, Phys. Rev. D89, 114006 (2014), 1403.4788

[17] J.R. Christiansen, S. Prestel (2015), 1510.01517

[18] S. Gieseke, T. Kasprzik, J.H. Kühn, Eur. Phys. J. C74, 2988 (2014), 1401.3964

[19] S. Kallweit, J.M. Lindert, P. Maierhöfer, S. Pozzorini, M. Schönherr, JHEP 04, 012 (2015), 1412.5157

[20] S. Frixione, V. Hirschi, D. Pagani, H.S. Shao, M. Zaro, JHEP 06, 184 (2015), 1504 . 03446

[21] S. Kallweit, J.M. Lindert, S. Pozzorini, M. Schönherr, P. Maierhöfer (2015), 1511.08692

[22] S. Frixione, B.R. Webber, JHEP 06, 029 (2002), hep-ph/0204244

[23] P. Nason, JHEP 11, 040 (2004), hep-ph/0409146

[24] S. Frixione, P. Nason, G. Ridolfi, JHEP 09, 126 (2007), 0707 . 3088

[25] S. Höche, F. Krauss, M. Schönherr, F. Siegert, Phys. Rev. Lett. 110, 052001 (2013), 1201.5882

[26] S. Höche, M. Schönherr, Phys. Rev. D86, 094042 (2012), 1208. 2815

[27] F. Cascioli, P. Maierhöfer, N. Moretti, S. Pozzorini, F. Siegert, Phys. Lett. B734, 210 (2014), 1309.5912

[28] S. Höche, F. Krauss, M. Schönherr, F. Siegert, JHEP 04, 024 (2011), 1008.5399

[29] M. Czakon, H.B. Hartanto, M. Kraus, M. Worek, JHEP 06, 033 (2015), 1502 . 00925

[30] L. Lönnblad, S. Prestel, JHEP 03, 166 (2013), 1211.7278

[31] K. Hamilton, P. Nason, E. Re, G. Zanderighi, JHEP 10, 222 (2013), 1309. 0017

[32] S. Höche, Y. Li, S. Prestel, Phys. Rev. D90, 054011 (2014), 1407. 3773

[33] S. Catani, F. Krauss, R. Kuhn, B.R. Webber, JHEP 11, 063 (2001), hep-ph/0109231

[34] K. Hamilton, P. Nason, G. Zanderighi, JHEP 10, 155 (2012), 1206.3572

[35] K. Hamilton, P. Nason, C. Oleari, G. Zanderighi, JHEP 05, 082 (2013), 1212 . 4504

[36] A. Karlberg, E. Re, G. Zanderighi, JHEP 09, 134 (2014), 1407 . 2940

[37] S. Höche, Y. Li, S. Prestel, Phys. Rev. D91, 074015 (2015), 1405. 3607

[38] R. Frederix, S. Frixione, JHEP 12, 061 (2012), 1209. 6215

[39] S. Höche, F. Krauss, P. Maierhöfer, S. Pozzorini, M. Schönherr, F. Siegert, Phys. Lett. B748, 74 (2015), 1402.6293

[40] N. Lavesson, L. Lonnblad, JHEP 12, 070 (2008), 0811. 2912

[41] S. Höche, F. Krauss, M. Schönherr, F. Siegert, JHEP 04, 027 (2013), 1207 . 5030

[42] T. Gehrmann, S. Höche, F. Krauss, M. Schönherr, F. Siegert, JHEP 01, 144 (2013), 1207.5031

[43] S. Höche, F. Krauss, M. Schönherr, F. Siegert, JHEP 08, 123 (2011), 1009. 1127

[44] F. Cascioli, S. Höche, F. Krauss, P. Maierhöfer, S. Pozzorini, F. Siegert, JHEP 01, 046 (2014), 1309.0500

[45] S. Höche, F. Krauss, M. Schönherr, Phys. Rev. D90, 014012 (2014), 1401.7971

[46] S. Höche, F. Krauss, S. Pozzorini, M. Schönherr, J.M. Thompson, K.C. Zapp, Phys. Rev. D89, 093015 (2014), 1403.7516

[47] M. Buschmann, D. Goncalves, S. Kuttimalai, M. Schönherr, F. Krauss, T. Plehn, JHEP 02, 038 (2015), 1410.5806 